\title{
A Comparative Study of Clinical Outcome of Clopidogrel and Prasugrel in Patients of Acute Coronary Syndrome Undergoing Percutaneous Coronary Intervention in Indian Settings
}

\author{
Authors \\ Dr Aditya, Dr Sharma A, Dr Kumar B \\ Corresponding Author \\ Dr Aditya \\ Swami Rama Himalayan University, \\ Email: adityasarin02@gmail.com
}

Medicine Department, Doiwala, Jolly Grant, Dehradun, Uttarakhand 248140 India

\begin{abstract}
Objective: The aim of this study is to see and compare the effect of two combinations of dual antiplatelet therapy (aspirin plus clopidogrel with aspirin plus prasugrel) that includes event rates of stent thrombosis, re-infarction, revascularization and bleeding in patient of acute coronary syndrome undergoing PCI.

Method: 223 patients of ACS attending cardiology OPD and emergency department were enrolled. Subjects were recruited through emergency presenting with a primary diagnosis of Acute Coronary Syndrome, after obtaining clearance from ethical and research committee and written informed consent from the patients.

Results: Though our study supports the idea of increased incidence in bleeding with prasugrel then clopidogrel group. This has also been validated by various studies that were carried in this regard $(15,16)$. In our study we didn't find any evidence of re-stenosis, re-infarction, Re-vascularization and mortality at 1,3,6,12 month follow up in both groups.

Conclusion: Despite less number of patient in prasugrel group the number of major and minor bleeding events were higher as compared to clopidogrel group with more number of patients, it can't be concluded with certainty that the complications can be high in prasugrel group. Although prasugrel a more potent antiplatelet drug as compared to clopidogrel ideally benefit should be more as per the previous studies but due to its cost, availability and lack of long term follow up clopidogrel can be still a preferred drug in these groups of patient.
\end{abstract}

\section{INTRODUCTION}

Throughout the world, there are more than 4 million people that suffer from NSTEMIs and 3 million have ST elevation MIs (STEMI) each year ${ }^{(1)}$. STEMIs is twice more prevalent amongst men as compared to women ${ }^{(2)}$. Women present more atypically as compared to men out of $30 \%$ atypical presentation ${ }^{(3)}$. In the United States about one million people have MI each year ${ }^{(4)}$. The risk of death in STEMI is about $10 \%$ in the developed world ${ }^{(5)} .5 \%$ have had an MI with little or no history of symptoms especially those who are over 75 years old ${ }^{(6)}$. Globally rates of MI are decreasing in period between 1990 and $2010^{(7)}$. 
Myocardial infarction (MI), used when there is evidence of myocardial necrosis in a clinical setting consistent with ischemia. Detection of rise or fall of cardiac biomarkers with at least one value above the $99^{\text {th }}$ percentile of upper reference limit and with any one of the mentioned: symptoms of ischaemia, new ST-T changes or new left bundle branch block, development of pathological $q$ wave in the ECG, imaging evidence of new loss of viable myocardium or new regional wall motion abnormality, identification of an intracoronary thrombus by angiography or autopsy. Chest pain or discomfort which may travel into the shoulder, arm, back, neck, or jawbeing most common symptom. Often it is in the centre or left side of the chest and lasts for more than 20 minutes. Other symptoms may include shortness of breath, nausea, feeling faint, a cold sweat, or feeling tired ${ }^{(8)}$.

Coronary artery disease is the most common cause of MI. High blood pressure, high cholesterol, diabetes, lack of exercise, obesity, smoking, poor diet, and excessive alcohol, are the risk factors generally found in association with an MI patient ${ }^{(9)}$. The mechanism of MI often involves the rupture of an atherosclerotic plaque leading to complete blockage of a coronary artery ${ }^{(4)}$. MIs are also caused by spasms of coronary artery which may be due to cocaine, emotional stress and extreme cold ${ }^{(10)}$. A wide variety of tests are now available for diagnosis including electrocardiograms (ECGs), blood tests, and coronary angiography. An ECG may confirm an ST elevation MI if ST elevation is present. Blood tests such as troponin $\mathrm{I} /$ troponin $\mathrm{T}$ and creatine kinase $\mathrm{MB}$ are employed out of which troponin carries higher sensitivity and specificity for diagnosis of myocardial infarction (11). Following an MI, serious complications such as heart failure, arrhythmias or cardiac arrest further can cause increase in mortality amongst these patient.

Aspirin a time tested drug is being used both in prevention and treatment of MI.

Nitroglycerin or opioids can be used to relieve chest pain; however, they do not improve overall outcomes. Supplemental oxygen should be used in those with low oxygen levels or those who have shortness of breath at the time of presentation ${ }^{(12)}$.

In STEMIs treatment which attempt to restore blood flow to the heart are typically recommended such as angioplasty, where the arteries are visualized with the help of contrast and pushed open with either balloon or stent placement or thrombolysis where mainstay of the treatment being pharmacotherapy based removal of the blockage ${ }^{(5)}$. Dual antiplatelet therapy is given to every patient who undergoes angioplasty either with bare metal stent or drug eluting stent. The therapy is to be given for a minimum of one year. People who have a non-ST elevation myocardial infarction (NSTEMI) which also includes unstable angina and patients are oftenly managed with heparin, with the additional use of angioplasty in those at high risk ${ }^{(12)}$. Bypass surgery $(\mathrm{CABG})$ may be recommended in blockages of multiple coronary arteries and diabetes rather than angioplasty (13). After an MI, lifestyle modifications along with long term treatment with aspirin, beta blockers, and statins is typically recommended.

\section{AIMS AND OBJECTIVES}

The aim of this study is to see and comparethe effect of two combinations of dual antiplatelet therapy (aspirin plus clopidogrel with aspirin plus prasugrel) that includes event rates of stent thrombosis, re-infarction, revascularization and bleeding in patient of acute coronary syndrome undergoing PCI.

\section{MATERIAL AND METHODS}

The study was conducted over a period of 12 months in 750 bedded tertiary care centre of north India. Subjects were recruited through emergency presenting with a primary diagnosis of Acute Coronary Syndrome, after obtaining clearance from ethical and research committee and written informed consent from the patients.

\section{Study Design:}

Type of the study: Non randomized interventional cohort study. 
Samples size: 223 patients of ACS attending cardiology OPD and emergency department were enrolled.

\section{Selection of Subjects:}

Inclusion Criteria: All patients of more than 18 years age belonging to either gender presenting as ACS undergoing PCI having following clinical presentation were included in the groups:

Patients with STEMI:

- $\leq 12$ hours after the onset of symptoms if primary PCI was planned

- $\leq 14$ days after receiving medical treatment for STEMI

Drugs were administered to the patient as soon as possible and before deciding medical / percutaneous intervention depending on consultant's choice and patient's consent.

Exclusion Criteria The following cases were excluded from the study:

-Age more than 65 years

-Any bleeding disorder

-Anaemia

-Thrombocytopenia

-Recent upper/ lower G.I. bleeding, recentstroke(less than 3 months)

-History of previous angiography

-History of previous PCI

-History of coronary artery bypass graft

After enrolment patients were put either of the group depending on their choice of selection of the drug along with aspirin (300mg loading followed by 75 to $150 \mathrm{mg}$ maintenance dose) in both the study groups:

Group A ( $\mathrm{n}=173)$ : Clopidogrel (300mg Loading dose followed by $75 \mathrm{mg}$ maintenance dose).

Group $\mathrm{B}(\mathrm{n}=50)$ :Prasugrel (60 $\mathrm{mg}$ loading dose followed by $10 \mathrm{mg}$ maintenance dose).

Study Tool: Structured study instruments (questionnaires/ subject proformas) was developed and were used to generate data.

Study Protocol: History and thorough clinical examination was done of all patient enrolled as per the inclusion and exclusion criteria. Relevant investigations example complete hemogram/ serum creatinine/ total bilirubin/ ALT/ AST/ HDL/ LDL/ Triglycerides/ RBS/ CPK-MB/
Troponin-I/ ECG/ ECHO and angiography were done and TIMI score was calculated in all patients. All these patient were followed up after 4 weeks, 3 month, 6 month and 12 months. Haemoglobin, haematocrit and platelet count was done during the follow up beside relevant history and clinical examination. Stent related complication was defined according to ARC classification

A. Any new episode of MI / Ischemia / Need for revascularization:

Both clinical and stress test (treadmill stress test (TMT) or stress 2D ECHO) was done to look for ischemia and need for revascularization was planned accordingly.

B. Bleeding events:

- Major Bleeding:

- Any intracranial bleeding(excluding micro hemorrhages $<10 \mathrm{~mm}$ )

- Clinically overt signs of associated with a drop in hemoglobin of $\geq 5 \mathrm{~g} / \mathrm{dl}$ or $\mathrm{a} \geq 15 \%$ absolute decrease in haematocrit

- Fatal bleeding (bleeding that directly results in death within 7 days)

- Minor Bleeding:

- Clinically overt (including imaging), resulting in hemoglobin drop of 3 to $<5$ $\mathrm{g} / \mathrm{dl}$ or $\geq 10 \%$ decrease in haematocrit

○ No observed blood loss: $\geq 4 \mathrm{~g} / \mathrm{dl}$ decrease in the haemoglobin concentration or $\geq 12 \%$ decrease in haemaotcrit

- Any overt sign of haemorrhage that meets one of the following criteria and does not meet criteria for a major or minor bleeding event, as defined above:

$>$ Requiring intervention

$>$ Leading to or prolonging hospitalization

- Prompting evaluation (leading to an unscheduled visit to a healthcare professional and diagnostic testing, either laboratory or imaging)

- Minimal bleeding:

- Any overt bleeding event that does not meet the above criteria 
○ Any clinically overt sign of haemorrhage (including imaging) associated with a $<3 \mathrm{~g} / \mathrm{dl}$ decrease in haemoglobin concentration or $<9 \%$ decrease in haematocrit

C. Mortality.

Patient dying due to myocardial infarction (M.I.) or history suggestive of MI like sudden cardiac arrest.

\section{Data Management and Statistical Analysis}

Data was analysed by using statistical software SPSS 22. Qualitative variables (Diabetes mellitus, hypertension etc) were represented in form of frequency and percentage. Quantitative data (BMI, BP, $\mathrm{Hb}$ etc) were represented in form of mean + standard deviation. Base line characteristics between two groups was compared with the help of Chi-square test and independent $t$ test. Repeated measure ANOVA was used to compare the mean of quantitative variable at different time intervals. Chi-square test was used to check association between qualitative variable. All standard test are tested at 5\% level of significance $\quad(p<0.05$ was considered as statistically significant).

\section{RESULTS}

The present study was carried out on 223 (Group $A=173$ and group $B=50$ ) patients diagnosed with ACS. Most patients who presented to our hospital were diagnosed with ACS belonged to the age group between 51-60 years (35.26\%), followed by 60-64 years $(27.17 \%)$. Of which 144 patients $(83.20 \%)$ were males, and 29 patients $(16.76 \%)$ were females in Group A and 45 patients $(90 \%)$ were males, and 5 patients (10\%) were females in Group B. Both the groups are comparable in terms of Age, pulse rate, systolic and diastolic blood pressure and RBS except BMI. 36.41\% in group $\mathrm{A}$ and $10 \%$ in group $\mathrm{B}$ of the total patients were hypertensive. $17.91 \%$ patients in group $\mathrm{A}$ and $9 \%$ in group B were previously diagnosed with Diabetes mellitus. $42.77 \%$ patients in group A and $46 \%$ in group B were smokers, while $26.01 \%$ percent in group A and $22 \%$ in group B were alcoholic.Table No.1 Shows the mean age of the patients in our study was approximately 53 in both the groups. Mean systolic blood pressure was 134 $\mathrm{mm}$ of $\mathrm{Hg}$ in group $\mathrm{A}$ and $128 \mathrm{~mm} \mathrm{Hg}$ in group B while mean diastolic pressure was $80 \mathrm{~mm} \mathrm{Hg}$ in group A and $76 \mathrm{~mm} \mathrm{Hg}$ in Group B. Mean pulse rate was 87 per minute in group $\mathrm{A}$ and 88 per minute in group B. Mean random blood glucose levels were 135.Table 2 shows Mean hemoglobin was 13.21 in group A and 13.55 in group B, Total leucocyte count was 9.29 in group A and 10.26 in group B, platelet count was 226.99 in group A and 221.23 in group B mean hematocrit was 39.9 in group $\mathrm{A}$ and 40.5 in group B. Mean Total Bilirubin was 1.2 in group A and 0.95 in group B, Mean ALT in group A was 42.5 and 30.62 in group B, Mean AST was 62.5 in group A and 38.56 in group $B$. Mean serum creatinine was 0.98 in group $\mathrm{A}$ and 1.16 in group $\mathrm{B}$, Mean sodium values are 138 in group $\mathrm{A}$ and 137 in group $\mathrm{B}$, mean potassium in group $\mathrm{A}$ is 4.28 and 4.25 in group B.Mean HDL was found to be less than 40, which favours at atherosclerosis in both groups. Mean LDL was levels were found be 131.08 in group A and 129 in group B, Triglycerides were 163.5 in group A and 170 in group B.Median value of CPK was calculated which was 94 in group A and 50 in group B.Mean Hemoglobin and platelet count were followed up of post PTCA patients at 1 month, 3 month, 6 month and 12 month in group A and group B no significant change in value was obtained. Table 3Mean Hematocrit report on follow up of post PTCA patients at 1 month 3 month and 6 month in group A and group B. Table 4 In our study we found 2 patients with minimal bleeding at 1 month follow up and 1 patient with minimal bleeding at 6 month follow up in group A. There was 1 patient with major bleeding 1 month follow up and 1 patient with minor bleeding at 1 month follow up, patient will minimal bleeding at 3 months and 1 patient with minimal bleeding at 6 month follow up in group B. 
Table No.1: Demographic and characteristics details

\begin{tabular}{|l|c|c|c|}
\hline Parameter & Group A $(\mathrm{n}=173)$ & Group B $(\mathrm{n}=50)$ & P value \\
\hline Age & $53.82 \pm 8.94$ & $53.72 \pm 8.14$ & 0.934 \\
\hline Body Mass Index & $25.66 \pm 2.69$ & $24.74 \pm 3.46$ & 0.047 \\
\hline Systolic Blood Pressure & $134.5 \pm 24.26$ & $127.82 \pm 18.05$ & 0.072 \\
\hline Diastolic Blood Pressure & $79.29 \pm 12.96$ & $75.68 \pm 11.09$ & 0.075 \\
\hline Pulse rate & $86.78 \pm 13.70$ & $87.42 \pm 13.04$ & 0.769 \\
\hline Random Blood Sugar & $135.64 \pm 74.35$ & $129.48 \pm 81.50$ & 0.614 \\
\hline
\end{tabular}

Table No: 2 Laboratory Investigations

\begin{tabular}{|l|c|c|c|}
\hline Parameters & Group A (n=173) & Group B (n=50) & p value \\
\hline Hemoglobin & $13.21 \pm 1.50$ & $13.55 \pm 1.53$ & 0.161 \\
\hline Total Leucocyte count & $9.29 \pm 3.05$ & $10.26 \pm 3.23$ & 0.051 \\
\hline Platelet Count & $226.99 \pm 72.61$ & $221.23 \pm 55.54$ & 0.604 \\
\hline Hematocrit & $39.78 \pm 4.50$ & $40.57 \pm 4.28$ & 0.270 \\
\hline Total bilirubin & $1.20 \pm 2.61$ & $0.95 \pm 0.37$ & 0.500 \\
\hline ALT & $42.59 \pm 77.07$ & $30.62 \pm 16.49$ & 0.277 \\
\hline AST & $62.54 \pm 83.73$ & $38.56 \pm 21.75$ & 0.046 \\
\hline S. Creatinine & $0.98 \pm 0.34$ & $1.16 \pm 0.62$ & 0.008 \\
\hline S. sodium & $138.01 \pm 4.39$ & $137.12 \pm 3.22$ & 0.184 \\
\hline S. potassium & $4.28 \pm 0.52$ & $4.25 \pm 0.46$ & 0.713 \\
\hline HDL & $36.83 \pm 9.12$ & $38.82 \pm 8.44$ & 0.168 \\
\hline LDL & $131.08 \pm 37.21$ & $128.98 \pm 30.37$ & 0.715 \\
\hline Triglyceride & $163.58 \pm 34.64$ & $170.2 \pm 39.81$ & 0.251 \\
\hline CPK-MB & 94 & 50 & \\
\hline
\end{tabular}

Table 3 Haematocrit values

\begin{tabular}{|l|c|c|c|}
\hline Parameter & Group A $(\mathrm{n}=173)$ & Group B $(\mathrm{n}=50)$ & P value \\
\hline Baseline & $39.78 \pm 4.50$ & $40.57 \pm 4.28$ & 0.270 \\
\hline Follow up at 1 month & $39.95 \pm 4.85$ & $40.89 \pm 4.29$ & 0.642 \\
\hline Follow up at 3 month & $40.67 \pm 3.87$ & $40.83 \pm 4.03$ & 0.798 \\
\hline Follow up at 6 month & $40.50 \pm 4.33$ & $41.65 \pm 3.96$ & 0.871 \\
\hline Follow up at 12 months & $42.52 \pm 2.14$ & $41.09 \pm 4.00$ & 0.664 \\
\hline
\end{tabular}

Table No.4: Bleeding event

\begin{tabular}{|l|c|c|c|c|c|c|}
\hline \multirow{2}{*}{ Parameter } & \multicolumn{2}{|c|}{ Major } & \multicolumn{2}{c|}{ Minor } & \multicolumn{2}{c|}{ Minimal } \\
\cline { 2 - 7 } & Group A & Group B & Group A & Group B & Group A & Group B \\
\hline Follow up at 1 month & 0 & 1 & 0 & 1 & 2 & 0 \\
\hline Follow up at 3 months & 0 & 0 & 0 & 0 & 0 & 2 \\
\hline Follow up at 6 months & 0 & 0 & 0 & 0 & 1 & 1 \\
\hline Follow up at 12 months & 0 & 0 & 0 & 0 & 0 & 0 \\
\hline
\end{tabular}




\section{DISCUSSION}

In this study, we found that the most number of patients presenting to our hospital were between age group of 51-60 years (35.26\%), followed by 60-64 years $(27.17 \%)$. Of which we found male predominance in our study who presented with typical symptoms then that of women. This predominance was observed in the previous studies ${ }^{(3)}$. Male predominance of acute coronary syndrome was observed in this study. Out of the 223 patients taken in this study, shows 144 patients $(83.20 \%)$ were males of the total 173 patients in Group A. and 45 patients (90\%) were males of the total 50 patients in Group B. Previous studies done to find the cause of male predominance of stroke incidence concluded that males had higher prevalence of current smoking, history of smoking and alcohol consumption. Others studiesalso found the incidence of dyslipidemia was higher in males as compared to the females which can progress atherosclerosis (14)

The primary complaint of most of the patients were chest pain, while a small majority of the patients came with sweating, palpitation and dyspnea.56.4\% of the patients presented within 25 days of onset of symptoms, while $29.14 \%$ presented within 1 days of onset of symptoms. Those presenting later usually belonged to remote areas of the state were they had poor transport facilities or had been admitted at some other hospital and were referred to our hospital for further management. Hypertension, the single most common modifiable risk factor for stroke was observed in 63 patients $(36.41 \%)$ out of the total 173 patients in group $A$ and 10 patients $(10 \%)$ in group B. Prevalence of hypertension increases with age. Prevalence of hypertension is about $45 \%$ at age $50,>60 \%$ at age 60 , and $>70 \%$ at age 70 . In Our study we enrolled the patients under 65 years.

$17.91 \%$ in group A and $18 \%$ in Group B of the patients enrolled in the study were previously diagnosed case of diabetes mellitus. Diabetes is a major risk factor for acute coronary syndrome as it causes lipid disturbances, progression of atherosclerosis. Prevalence of diabetes observed in our patients was similar to the previous studies which identified diabetes as a risk factor for atherosclerosis. They had an prevalence of $30 \%$ of diabetes mellitus in patients of acute coronary syndrome while our study had prevalence of $17 \%$ (9).

$42.77 \%$ of the patients in group A and $46 \%$ in group B enrolled in the study were smokers. Increased risk of stroke in smokers can be attributed to the fact that smoking leading to hypertension, which itself is a risk factor for stroke. Smoking also decreases the levels of HDL in the blood and increases the levels of LDL which favour atherosclerosis. $30 \%$ of the patients had consumption of alcohol intake in moderate amount.

Demographic parameters revealed the mean systolic blood pressure for the enrolled patients were $134 \mathrm{~mm}$ of $\mathrm{Hg}$ and $127 \mathrm{~mm}$ of $\mathrm{Hg}$ in group a and group $b$ respectively. Many of the patients (38\%), were diagnosed cases of hypertension, on regular antihypertensive. Mean Diastolic blood pressure was $80 \mathrm{~mm}$ of $\mathrm{Hg}$ in group A and $76 \mathrm{~mm}$ $\mathrm{Hg}$ in group B. Mean hemoglobin was 13.21 in group A and 13.55 in group B, Total leucocyte count was 9.29 in group A and 10.26 in group B, platelet count was 226.99 in group A and 221.23 in group B mean hematocrit was 39.9 in group A and 40.5 in group B. Mean Total Bilirubin was 1.2 in group A and 0.95 in group B, Mean ALT in group A was 42.5 and 30.62 in group B, Mean AST was 62.5 in group A and 38.56 in group B. Mean serum creatinine was 0.98 in group A and 1.16 in group $\mathrm{B}$, Mean sodium values are 138 in group $\mathrm{A}$ and 137 in group $\mathrm{B}$, mean potassium in group $\mathrm{A}$ is 4.28 and 4.25 in group B. Mean HDL was found to be less than 40 , which favours at atherosclerosis in both groups. Mean LDL was levels were found be 131.08 in group A and 129 in group B, Triglycerides were 163.5 in group A and 170 in group B.In our study we followed the patient with acute coronary syndrome who underwent angioplasty at 1,3,6 and 12 months in both the groups. Patient were followed with haemoglobin, haematocrit and platelet count over 
these months. There was no significant change in the follow up values of haemoglobin and platelet count when compared between two groups. There was no significant change in values of haemoglobin, haematocrit and platelet count in group A when followed up as well as in group B.

Two patients who were on clopidogrel developed minimal bleed at 1 month follow up and 1 patient at 6 month follow up. One patient on prasugrel developed major bleed within one month of initiation of therapy, one patient developed minor bleed at 1 month follow up and 2 patient developed minimal bleed at 2 month follow up.Though our study supports the idea of increased incidence in bleeding with prasugrel then clopidogrel group. This has also been validated by various studies that were carried in this regard ${ }^{(15,16)}$. In our study we didn't find any evidence of re-stenosis, re-infarction, Revascularization and mortality at 1,3,6,12 month follow up in both groups. Though study done by Wiviott SD, Braunwald E, McCabe $\mathrm{CH}$ et al reported less number of complications in the prasugrel group. Rates of myocardial infarction (9.7\% for clopidogrel vs. $7.4 \%$ for prasugrel; $\mathrm{P}<0.001$ ), urgent target-vessel revascularization (3.7\% vs. $2.5 \% ; \mathrm{P}<0.001)$, and stent thrombosis ( $2.4 \%$ vs. $1.1 \% ; \mathrm{P}<0.001)$. As our study showed slightly higher risk of bleeding in patient receiving prasugrel. A study showed major bleeding was observed in $2.4 \%$ of patients receiving prasugrel and in $1.8 \%$ of patients receiving clopidogrel (hazard ratio, $1.32 ; 95 \% \mathrm{CI}, 1.03$ to $1.68 ; \mathrm{P}=$ $0.03)$. Also greater in the prasugrel group was the rate of life-threatening bleeding $(1.4 \%$ vs. $0.9 \%$; $\mathrm{P}$ $=0.01)$, including nonfatal bleeding $(1.1 \%$ vs. $0.9 \%$; hazard ratio, $1.25 ; \mathrm{P}=0.23$ ) and fatal bleeding $(0.4 \% \text { vs. } 0.1 \% ; \mathrm{P}=0.002)^{(16)}$

\section{CONCLUSION}

There was no evidence of restenosis, re-infarction, revascularization in both the groups. There was no evidence of mortality in the study in both the groups. Despite less number of patient in prasugrel group the number of major and minor bleeding events were higher as compared to clopidogrel group with more number of patients, it can't be concluded with certainty that the complications can be high in prasugrel group. Although prasugrel a more potent antiplatelet drug as compared to clopidogrel ideally benefit should be more as per the previous studies but due to its cost, availability and lack of long term follow up clopidogrel can be still a preferred drug in these groups of patient.

\section{REFERENCES}

1. White HD, Chew DP; Chew "Acute myocardial infarction. Lancet 372 (9638): 570-84.

2. O'Gara PT, Kushner FG, Ascheim DD, et al. "ACCF/AHA guideline for the management of ST-elevation myocardial infarction: a report of the American College of Cardiology Foundation/ American Heart Association Task Force on Practice Guidelines." Circulation 2013; 127 (4): 362-425.

3. Coventry LL, Finn J, Bremner AP. Sex differences in symptom presentation in acute myocardial infarction: a systematic review and meta-analysis. Heart \& lung Journal of Critical Care 2011; 40: 477-91

4. "What Is a Heart Attack?". Nhlbi.nih.gov. National Heart, Lung, and Blood Institute (NHLBI) - NHLBI, NIH [Internet]. 2013 [cited 17 december 2013]. Available from: http://www.nhlbi.nih.gov.

5. Steg PG, James SK, Atar D. ESC Guidelines for the management of acute myocardial infarction in patients presenting with ST-segment elevation. European Heart Journal 2011; 33: 2569619.

6. Valensi P, Lorgis L, Cottin Y. Prevalence, incidence, predictive factors and prognosis of silent myocardial infarction: a review of the literature. Arch Cardiovasc Dis 2011; 10:178-88.

7. Moran AE, Forouzanfar MH, Roth GA et al. "The global burden of ischemic heart disease in 1990 and 2010: the Global 
Burden of Disease 2010 study." . Circulation 2010; 129 (14): 1493-501.

8. Thygesen, K., Alpert, J. S., Jaffe, A. S., Simoons, M. L., Chaitman, B. R. And White, H. Det al. "Third Universal Definition Of Myocardial Infarction". Circulation 126.16 (2012): 2020-2035. Web. 23 Dec. 2015.

9. Mehta PK, Wei J, Wenger NK. Ischemic heart disease in women: A focus on risk factors. Trends in Cardiovascular Medicine 2014; 25 (2): 140-151.

10. "What Causes a Heart Attack?". Nhlbi.nih.gov. National Heart, Lung, and Blood Institute (NHLBI) - NHLBI, NIH [Internet]. 2013 [cited 17 december 2013]. Available from: http://www.nhlbi.nih.gov.

11. "How Is a Heart Attack Diagnosed?". Nhlbi.nih.gov. National Heart, Lung, and Blood Institute (NHLBI) - NHLBI, NIH [Internet]. 2015 [cited 17 december 2013]. Available from: http://www.nhlbi.nih.gov.

12. O'Connor, RE; Brady W; Brooks SC. Part 10: acute coronary syndromes: American Heart Association Guidelines for Cardiopulmonary Resuscitation and Emergency Cardiovascular Care. Circulation 2012;122: S787-817.

13. Hamm CW, Bassand JP, Agewall S. ESC Guidelines for the management of acute coronary syndromes in patients presenting without persistent ST-segment elevation: The Task Force for the management of acute coronary syndromes (ACS) in patients presenting without persistent STsegment elevation of the European Society of Cardiology (ESC). Eur. Heart J. 201032 (23): 2999-3054.

14. McDaniel MC, Willis P, Walker B, et al. Plaque necrotic core content is greater immediately distal to bifurcations compared to bifurcations in the proximal lad of patients with CAD. Am J Cardiol. 2008; 102(8): $242 \mathrm{i}$.
15. Wiviott SD, Trenk D, Frelinger AL. Prasugrel compared with high loading- and maintenance-dose clopidogrel in patients with planned percutaneous coronary intervention: the Prasugrel in Comparison to Clopidogrel for Inhibition of Platelet Activation and Aggregation-Thrombolysis in Myocardial Infarction trial. Circulation 2007;116:2923-32.

16. Wiviott SD, Braunwald E, McCabe $\mathrm{CH}$. Prasugrel versus clopidogrel in patients with acute coronary syndromes. N Engl J Med 2007;357:2001-15. 